\title{
Formación postdoctoral y producción científica
}

\section{Postdoctoral training and scientific production}

\author{
*Concepción Toledo, D.N. ${ }^{1}$; Miño Valdés, J.E. ${ }^{2}$; González Suárez, E. ${ }^{3}$ \\ ${ }^{1}$ Facultad de Ciencias Sociales, Universidad Central de Las Villas. Cuba \\ ${ }^{2}$ Dpto.de Física, Facultad de Ingeniería, Universidad Nacional de Misiones. \\ Argentina \\ ${ }^{3}$ Dpto. de Ingeniería Química, Facultad de Química y Farmacia, Universidad
} Central de Las Villas, Cuba

\section{RESUMEN}

En este trabajo se presentan las razones que justifican la inclusión de actividades postdoctorales en los Departamentos de Ingeniería Química de la Universidad. La continua labor científica en: actualización, innovación, transferencia y publicación de conocimientos, pretende que los doctores aumenten su producción científica mediante el trabajo colaborativo. Con una visión prospectiva, se busca aumentar la preparación de recursos humanos para el relevo generacional. Se explican fundamentos básicos de Planes Postdoctorales y se incluye como ejemplo: el caso de un docente investigador de una Universidad Argentina, en el contexto de la colaboración sur-sur. Finalmente se fundamenta la estrategia de formación continua de la actividad posdoctoral, como contribución a la formación de líderes científicos para la Universidad o la Industria Química y Fermentativa.

Palabras clave: formación postdoctoral, producción científica.

\section{ABSTRACT}

In this paper the reasons for the inclusion of postdoctoral activities in the departments of Chemical Engineering at the University are presented. The ongoing scientific work: renovation, innovation, knowledge transfer and publication are intended that doctors increase their scientific production through collaborative work. With a prospective vision, it seeks to increase the training of human resources for generational change. Postdoctoral basics are explained Plans and is included as an example: for a researcher teacher University of Argentina, in the context of south-south cooperation. Finally the lifelong learning strategy of the postdoctoral activity is based, as a contribution to the formation of scientific leaders for college or the chemical and fermentation industry.

Keywords: postdoctoral training, scientific production.

\section{INTRODUCCION}

Una acción estratégica de la universidad es el crecimiento sistemático y continuo de sus recursos humanos más calificados. La obligación de los líderes científicos es mantener la fuerza de tracción sobre los colectivos docentes, y a través de ellos, trasmitir a los más jóvenes la necesidad de trabajar en las

\footnotetext{
*Autor Correspondiente: Concepción Toledo D.N. Facultad de Ciencias Sociales, Universidad Central de Las Villas. Carretera de Camajuani km 5,5 Santa Clara, Cuba E-mail: dianac@uclv.edu.cu Fecha de recepción: setiembre 2016; Fecha de aceptación: octubre 2016
} 
demandas de la sociedad y de la industria de procesos químicos y fermentativos.

No entretenerse en el éxtasis de los "oasis científicos", ni frenarse por los obstáculos que se presenten en ese esfuerzo por incrementar el impacto del conocimiento colectivo, en beneficio de una región del país.

Es un requerimiento de los promotores y líderes científicos universitarios el reconocimiento profesional del mundo empresarial y la visibilidad científica.

Lo anterior, fundamenta la necesidad de elaborar una estrategia de incremento de las capacidades, mediante sistemas de postgrado (Maestrías, Doctorados y Postdoctorados) de generación, asimilación de tecnologías y de obtención de productos químicos de alto valor agregado.

No obstante el esfuerzo en la actividad de formación de doctores, se ha realizado en el contexto latinoamericano, dos fenómenos negativos se han presentado:

a) el impacto de la investigación científica en la industria química y fermentativa no ha mantenido la dinámica requerida para la economía latinoamericana.

b) muchos doctores, que en su momento constituyeron el núcleo duro de los investigadores científicos, han reducido su actividad científica una vez defendida su tesis doctoral.

Esto último se ha manifestado de forma perjudicial en la Educación Superior por la insuficiente formación de nuevos doctores, con un papel meramente formal en la pirámide científica.

El empuje de los doctores más jóvenes avanza, no por el relevo natural de generaciones, sino por la falta de dedicación sistemática a la creatividad investigativa, lo que se manifiesta como interrupciones perjudiciales a la continuidad del liderazgo científico, reconocimiento profesional y la visibilidad científica, en los centros de investigación científicas, base de la excelencia de la educación superior.

Los factores que explican este estancamiento en el desarrollo científico de valiosos compañeros ha sido entre otros, la asignación de tareas ajenas a la investigación y creación científica que los ha alejado de esa actividad, también porque no decirlo, la falta de resistencia para mantener el necesario ritmo creativo investigativo y la carencia de una figura académica y de formación que obligue a superar las dos causas anteriores.

Por ello el Departamento de Ingeniería la Universidad Central de Las Villas se ha trazado una estrategia de formación posdoctoral contínua que tiene como objetivos incrementar:

- La competitividad de los doctores de menos desempeño científico.

- El impacto en la formación de capacidades de investigación y desarrollo de los doctores de largo y fructífero desempeño.

- La capacidad experimental en el Dpto. de Ingeniería Química de la Facultad.

- La actualización científica de los doctores que se han alejado del quehacer científico investigativo $o$ que no han encontrado la continuidad.

- La producción de conocimientos en Ingeniería Química para superar las limitaciones económicas de la agroindustria en los países del sur.

- Estrechar los lazos de colaboración entre Universidades del sur con vistas a gerenciar conocimientos para impulsar su desarrollo. 
- Garantizar el legado científico de los docentes e investigadores de mayor experiencia y desempeño para el relevo científico generacional de cada país.

En el cumplimiento de estos objetivos se considera la colaboración con el sector empresarial como una vía idónea para aplicar conocimientos de forma conjunta en función de las demandas tecnológicas de la industria de procesos químicos y fermentativos.

Se busca garantizar a través de su introducción un rápido impacto de los resultados de las investigaciones, pues se concibe el proceso del desarrollo científico desde la idea novedosa del investigador, hasta que la misma se convierte en un producto que va al mercado a enfrentar el reto de la competitividad.

Es necesario fundamentar soluciones que garantizan el aporte al conocimiento de la investigación y con ello avalan las defensas de grados científicos.

\section{DESARROLLO}

\section{Fundamentos del Vínculo: Centros de Generación de Conocimientos- Empresas.}

El impacto de la Investigación el Desarrollo y la innovación tecnológica $(\mathrm{I}+\mathrm{D}+\mathrm{i})$ han generado cambios duraderos en la economía, la ciencia, la tecnología y el medio ambiente. Esto se debe a la introducción de valor agregado a los productos, servicios, procesos y tecnologías, manifestándose además en el surgimiento y consolidación de determinados sistemas tecnológicos que al actuar en conjunto expanden su acción hasta producir una verdadera modificación en los patrones y en el comportamiento de la economía y la sociedad.

Por otro lado, si bien es una realidad, que las instituciones académicas no son los únicos centros de producción de conocimientos, lo que sí se afirma es que la Educación Superior es el elemento socio-institucional básico de producción para los trabajadores del conocimiento.

Ha cobrado cada vez más importancia el papel de las instituciones de la Educación Superior en la transferencia de conocimientos y tecnología hacia la producción y la sociedad (Waissbluth, 1994).

Así pues la actividad de una empresa y los Centros de la Educación Superior están muy vinculadas a la ciencia, la tecnología, el mercado y la sociedad, siendo esta la que realmente se satisface con los productos que se generan con los resultados de la producción científica.

Se conseguirán resultados impactantes en las economías de los países cuando se logre que la propia concepción, planificación y ejecución del trabajo científico esté orientado a la culminación del ciclo completo de la actividad científico productiva.

Una investigación a ciclo completo llega a feliz término con la introducción de su producto científico, siempre y cuando realmente esta se desarrolla sobre la base de un interés colectivo por parte de todos los factores para desarrollar el producto que demanda el mercado.

En esta colaboración de las empresas de producción y servicios y los centros de generación de conocimientos, no se debe dejar de considerar las características específicas de cada una de ellas.

En este análisis es necesario considerar los ámbitos de la actividad de una empresa y de los Centros de la Educación Superior. Realmente los ámbitos de 
las empresas y de los Centros de la Educación Superior son diferentes, como se observa en la Figura 1, las áreas de acción de las empresas están enmarcadas en la Ciencia, la Tecnología y el Mercado, siendo el alcance de la Educación Superior más amplio al incluir su estrecho vínculo con la sociedad (Cunningham, 2002).
CIENCIA
TECNOLOGÍA
MERCADO
SOCIEDAD

\section{EMPRESAS}

\section{CENTROS DE EDUCACIÓN SUPERIOR}

Figura 1. Ámbitos de las Empresas y los Centros de la Educación Superior

La cooperación tecnológica representa en la actualidad una estrategia competitiva que permite no solo a las empresas avanzar conjuntamente en el desafío tecnológico mediante la gestión adecuada de las alianzas tecnológicas con los centros de generación de conocimientos, sino también a los Centros de Generación de Conocimientos en el fortalecimiento de la formación de su personal con una visión de investigación a ciclo completo. Esto permite retroalimentar las demandas y desafíos del conocimiento revelando el camino de la formación de las nuevas generaciones de profesionales y científicos.

De hecho tanto para las organizaciones empresariales como para las generadoras de conocimientos, la capacidad de observación y aprendizaje continuo son destrezas fundamentales, para el éxito y donde la institución, la capacidad de anticipación y la velocidad de respuesta determinan la supervivencia de la propia organización.

Por ello las organizaciones que generan conocimientos deben considerar, para la elaboración de su Política Científica entre otras cosas, que no es aconsejable trabajar en temas de investigación científica que no resulten en una contribución al propio desarrollo de las instituciones de Generación de conocimientos, lo que se mide en primer término en la formación de recursos humanos.

De todo lo anterior se infiere que el camino necesario parta llevar adelante de forma fructífera el trabajo investigativo en aras de lograr un ciclo completo y de introducir los resultados del trabajo científico lo constituyen las alianzas estratégicas con el sector empresarial para su desarrollo prospectivo, lo que permitirá esbozar también las demandas de la política científica, al considerar además y junto con ello la dialéctica del desarrollo de las ciencias mismas previendo las mejores alternativas de generación de conocimientos en la formación de las generaciones futuras.

Por lo que es necesario que los proyectos de investigación se formulen como parte de oportunidades de negocios de las empresas, identificados muchas veces por Empresas y Centros de Generación de Conocimientos (CGC), debido al interés de introducir los resultados científicos, que con una actividad investigativa proactiva intensa, se pueden lograr más temprano que tarde. Esta aplicación logra el incremento prospectivo de la competitividad del CGC a través de un crecimiento sistemático en la formación científica, vista multilateralmente de los investigadores participantes, todo ello, en el concepto de que se requiere cada días más acortar el tiempo entre la investigación, la innovación y el producto final. 
Entonces, ha sido necesario, con visión de futuro trazar una estrategia de investigación a largo plazo que permita asegurar el desarrollo sostenido del Departamento de Ingeniería Química y su aliado estratégico el Centro de Análisis de Procesos a través de la línea científica universitaria de Estrategias y Tecnologías para la obtención de productos químicos de alto valor agregado.

\section{Estrategia de investigación para obtener productos químicos de alto valor agregado}

Cuando se decide gestionar el desarrollo científico de un CGC en estrecho vínculo con las demandas del sector productivo y la sociedad en su conjunto, una alternativa es organizar la labor investigativa a través de lo que se conoce como Problemas Económico-Sociales, a los que el trabajo científico de la universidad debe contribuir a solucionar.

Como una expresión de la demanda del Conocimiento que la sociedad y dentro de ella la producción plantea como retos a los CGC y colectivos de Trabajo Científico en las que según los diferentes sectores del conocimiento organizan sus tareas los investigadores.

Le corresponde al CGC la coordinación de la Línea Científica para la obtención de productos químicos de alto valor agregado.

Por otro lado, en los procesos de transferencia y asimilación de tecnologías siempre habrá incertidumbre y cuanto mayor sea el nivel de incertidumbre, mayor será la resistencia que ofrezcan los agentes afectados de una u otra forma por los cambios tecnológicos, y mayor será también el esfuerzo necesario para realizar el proceso hasta el final. (González, 2005)

La investigación científica es una función inherente de la Educación Superior por lo que es entonces altamente deseable que se optimicen también las Estrategias de Capacidades Investigativas y de desarrollo de los docentes, lo que indudablemente puede también lograrse a partir de:

- Un adecuado conocimiento y valoración de las tendencias de desarrollo universal sobre una determinada temática.

- Una ágil y efectiva estrategia de investigación experimental en retroalimentación con el estudio de escalado y factibilidad de los procesos tecnológicos, lo que requiere la ingenierización de la investigación.

Los aspectos claves en esta Estrategia han sido sin dudas:

- El vínculo con la demanda. Los estudios técnicos, económicos y ambientales. Los estudios previos inversionistas.

- Asimilar los conocimientos que se generan constantemente. Intercambio sistemático con científicos. Vigilancia Tecnológica.

- $\quad$ Gestión del conocimiento y desarrollo prospectivo.

- La minimización de los gastos de investigación. Diseño experimental y la modelación matemática.

- Formación y crecimiento de todos los recursos humanos. Combinación de la teoría, el debate científico y la práctica real.

\section{Incorporación de las actividades posdoctorales a la estrategia de fortalecimiento de las capacidades de desarrollo, científicas e investigativas}

Por su importancia en el trabajo futuro y específicamente en el relevo de generaciones, se estableció una vía para la creación simultánea de capacidades científicas y tecnológicas, partiendo de un grupo de ideas básicas sobre la formación posdoctoral, que son las siguientes: 
- Es requerimiento en la formación posdoctoral que la investigación desarrollada tenga aplicación práctica a corto, mediano o largo plazo, siendo en extremo eficaz que la génesis del trabajo de investigación sea una demanda real de la producción.

- Se debe organizar la respuesta a la demanda de conocimiento como un Proyecto de Investigación y Desarrollo con todos los requerimientos organizativos de esta actividad.

- $\quad$ Que el Coordinador del Proyecto debe tener una visión de generador de conocimientos, tutor científico e introductor de resultados.

- $\quad$ Se requiere de un trabajo en equipo y contar con un asistente del responsable encargado no solo del peso operativo de la ejecución del Proyecto, y sobre todo, de la ejecución del trabajo central del proyecto lo que debe redundar en su formación Posdoctoral según sea el caso, así mismo se incorporan al Proyecto otros investigadores en formación, como estudiantes de grado, maestría y doctorado.

- Las sesiones científicas sistemáticas para debatir los resultados y las estrategias de continuación de la labores del Proyecto, con la presencia del Coordinador del Proyecto, pueden ser organizadas por el asistente del proyecto, como una vía a forzar su desarrollo profesional personal de una manera más activa.

- La elaboración periódica de documentos científicos para ordenar las ideas y someterlos a la crítica de otros especialistas.

- La búsqueda sistemática no solo de la solución de los problemas, sino también de los impactos científicos que garanticen la formación en este aspecto.

En esta dirección se deben realizar un conjunto de acciones como vías de lograr la viabilidad de la estrategia elaborada y la ejecución de las ideas básicas para la formación de investigadores científicos y su creciente potenciación de posibilidades, que incluyen:

- Favorecer el vínculo con el desarrollo de la industria química y fermentativa en la región de acción directa del CGC de los docentes involucrados.

- Incrementar la dinámica en la participación de las empresas y los centros de generación de conocimiento como redes estables de trabajo, no solo con personas sino también con ideas y formas de actuación.

- Favorecer la vinculación con los Parques Tecnológicos de la región como una vía de establecer oportunidades de terminación de resultados y de transferencia de conocimientos al sector productivo.

- Gestar Proyectos Internacionales, Binacionales y Nacionales con vistas a alcanzar nuevos conocimientos de transferencia al sector productivo.

Aquí un aspecto que queda como problema cardinal es el referido al crecimiento científico del asistente del Proyecto, ya sea un joven doctor en actividad posdoctoral por lo que su labor como asistente del Proyecto debe implicar:

- Profundización teórica sobre los métodos y fundamentos de la solución de la demanda real que se trata de resolver.

- Control operativo del Trabajo en equipo y del Proyecto en general.

- Representación externa e interna en la ejecución del proyecto en la presentación de resultados. 
- Colaboración internacional en las investigaciones con expertos de otros países para conocer directamente diferentes enfoques en el trabajo investigativo.

- $\quad$ Preparación de tareas de divulgación del Proyecto y sus resultados.

- $\quad$ Contacto directo con los introductores del resultado.

- La formulación de Proyectos de apoyo, con instituciones financistas de las labores de investigación nacionales e internacionales.

En la Política Científica para el período se han previsto como una necesidad, además de los proyectos de investigación que respondan a las demandas en el vínculo con la empresas, un proyecto para catalizar el autodesarrollo de la comunidad científica de la industria de procesos químicos en la temática de: Fortalecimiento de la Capacidad Científica en la Región Central de Cuba. (González; 2014)

\section{Requisitos y definiciones:}

Los requisitos para la realización de actividades posdoctorales son:

- Que se ejecute en el contexto de los proyectos aprobados de la línea de investigación.

- Que los resultados alcanzados sean transferibles a las empresas de producción a mediano o corto plazo y tengan desde el primer momento un usuario interesado.

- Que se incluya en el posdoctoral una acción de transferencia de tecnología.

- Que se incluya en el posdoctoral una acción de vigilancia tecnológica.

- Que se deriven de su ejecución propuestas de temas doctorales.

- Que tenga de duración no menos de 6 meses.

- Que se incluya un fuerte componente experimental.

- Que se utilicen métodos avanzados de procesamiento de datos.

- Que se obtengan publicaciones de impacto en la web.

- Que se tenga un tutor extranjero además del cubano.

- Que se incluya una estancia de no menos de 30 días en una institución extranjera, siempre como complemento del proyecto que responde a la demanda de la economía de Cuba

- Que tengan un impacto en el postgrado de la facultad.

- $\quad$ Que se concluya con un documento de proyección científica.

\section{Una experiencia sur-sur}

Antecedentes: las relaciones de trabajo científico entre la Universidad Nacional de Misiones, Argentina y la Universidad Central "Marta Abreu" Cuba datan del año 1995 y ha incluido varios Proyectos Científicos sobre el uso de la biomasa como fuente de productos químicos y un Proyecto de Formación de doctores de la universidad Argentina en los Programas Doctorales de la Universidad Cubana.

Los esfuerzos del proyecto destinado a la formación de doctores estuvieron encaminados a lograr resultados en la formación de científicos ( 8 en total) y a superar las limitaciones de tecnologías para viabilizar su transferencia a la actividad empresarial en la provincia de Misiones Argentina (González y Miño; 2013).

En este contexto se consideró adecuado elaborar una conceptualización de las experiencias científicas acumuladas como resultado de la colaboración en el desarrollo de las investigaciones conjuntas, encaminadas a un fortalecimiento de la Gestión de $\mathrm{I}+\mathrm{D}+\mathrm{i}$ en procesos químicos que utilizan la biomasa como materia prima. 
Para lograr este objetivo se diseñó una estancia (pasantía) Postdoctoral en Gerencia de la Ciencia y la Innovación para la industria química y fermentativa, para uno de los docentes argentinos que se había doctorado en el Proyecto conjunto de Formación de Doctores de la Universidad Nacional de Misiones, en la Universidad Central "Marta Abreu" de las Villas Cuba.

Durante su estancia posdoctoral y como resultado de la misma se logró la incorporación del docente/investigador Argentino (Ing. Qco., MSc. en Tecnología de Alimentos y Dr. en Ciencias Técnicas) a las actividades propias del colectivo científico del Dpto. de Ing. Química de la Universidad Central "Marta Abreu" de Las Villas, en la línea de investigación: Estrategia y Tecnologías de obtención de productos químicos de alto valor agregado, además se incorporo a las asesorías de tesis de investigación para la Maestría de Gerencia de la Ciencia e Innovación y del Programa Doctoral de Ingeniería Química de la misma Universidad Cubana.

Producto de una labor de conceptualización de los resultados científicos productos de la colaboración binacional se logró la publicación de 7 Obras Científicas tituladas:

1. "Aspectos de la Estrategia de Procesos para el Aprovechamiento de la Biomasa como fuente de productos químicos y biocombustibles". / González y Miño, (2014) / ww.editorial.unam.edu.ar / ISBN 978-950579-339-6.

2. "Acciones para la correcta terminación y valoración de resultados en la industria química y fermentativa".

González y Miño, (2014) / ww.editorial.unam.edu.ar / ISBN 978-950579-379-2.

3. "Estrategia Innovativa en el desarrollo de una Tecnología para Elaborar Vino Blanco con Uvas no Viníferas". / Miño et al, (2015) / ww.editorial.unam.edu.ar / ISBN 978-950-579-369-3.

4. "Vías para la Gestión de Conocimientos con apoyo de la comunidad científica para contribuir a la mitigación del impacto ambiental de la industria química y fermentativa". / González y Miño, 2015) / ww.editorial.unam.edu.ar / ISBN 978-950-579-368-6.

5. "Estrategia de Colaboración de la Comunidad Científica Sur-Sur para el Desarrollo de la Industria Química de Angola". / González y Miño, (2016) / ww.editorial.unam.edu.ar / ISBN 978-950-579-406-5.

6. "La formación en la Gestión de Ciencia e Innovación desde la Universidad para el Desarrollo Local". / Miño et al., (2016) / ww.editorial.unam.edu.ar / ISBN 978-950-579-409-6.

7. "Política Científica y Tecnológica para el Incremento de las Oportunidades de Negocios en Beneficio del Desarrollo Local". / Miño et al., (2016) / www.editorial.unam.edu.ar / ISBN 978-950-579-408-9.

Las publicaciones han sido debidamente depositadas en los centros de documentación de la Universidad Nacional de Misiones, Argentina, y la Universidad Central de las Villas, Cuba y Universidad de Camagüey, Cuba.

En adición, se logró la formulación de un Proyecto Binacional con sede en Latinoamérica sobre la obtención de biodiesel y el armado de una Maestría en Energía Renovable que dará continuidad a las relaciones de colaboración y la formación de científicos, entre Universidades sur-sur. 


\section{Probable impacto en la formación de líderes científicos en la formación postdoctoral}

Indudablemente, como se ha dicho, sin científicos no puede haber investigación científica, pero los científicos necesitan de líderes que estén capacitados para promover y dirigir el trabajo científico fruto de la experiencia. Las buenas políticas de desarrollo científico/tecnológico garantizan la consolidación del proceso de enseñanza-aprendizaje en todos los niveles educativos, para impactar finalmente en la vida socioeconómica del país.

Este accionar requiere de un espacio en el cual se materialicen la práctica de las ideas, para lo que la experiencia ha demostrado que las actividades posdoctorales son un marco adecuado para catalizar la continuidad de la formación de los doctores.

\section{CONCLUSIONES}

1. La continuidad de la escuela científica y la capacidad de formar profesionales de alta calidad, está determinado por la continuidad de la formación de promotores y lideres científicos con inserción y reconocimiento de la sociedad. Lo que se ha debilitado por diferentes causas en los últimos años.

2. La alianza Universidad-Empresa, es una alternativa en beneficio mutuo, para fortalecer los vínculos entre el sector generador de conocimientos y el de producción de bienes y servicios, la cual debe continuar perfeccionándose acorde con las demandas de la realidad de la sociedad.

3. La actividad de los Centros de Educación Superior está muy vinculada a la sociedad y por ella a la demanda del mercado, ejerciendo una fuerza inductora en la Política Científica y la formación de sus Recursos Humanos que conllevan a encontrar formas más actualizadas para crecimiento sistemático y continuo de la capacidad de desarrollo de la ciencia y la técnica.

4. Una investigación a ciclo completo llega a feliz término con la comercialización de su producto científico cuando realmente esta se desarrolla sobre la base de un interés colectivo por parte de todos los factores para desarrollar el producto que demanda el mercado. Además se resuelven todos los problemas, que permiten el impacto del conocimiento científico en satisfacer las demandas del mercado, lo que brinda infinitas posibilidades de hacer Ciencia con crecimiento de los Recursos Humanos en el plano científico y profesional.

5. La Producción conjunta de obras científicas que conceptualicen las diferentes experiencias de sus autores es un legado a las nuevas generaciones que permitirán consolidar las escuelas científicas.

\section{REFERENCIAS BIBLIOGRAFICAS}

Cunningham, R; Laborde, M. A. González, E.; (2002). La gestión de proyectos en la gerencia de conocimientos para el uso de la biomasa como fuente de productos químicos y energía. Experiencia y proyección. I. Encuentro Nacional e Internacional de Gestión Tecnológica. Del 15-21/mayo/02. Caracas, Venezuela.

González, E.; (2005). Vías para el diseño de nuevas instalaciones de la industria química, fermentativa y farmacéutica. Ed. Cient.Técn., La Habana, pp.263. ISBN 978-950-579368-6.

González Suárez, E. y Miño Valdés J.E.; (2013). Estrategia de cooperación internacional entre Universidades sur-sur. Orientadas a superar las limitaciones económicas de una tecnología. Editorial Universitaria de Misiones. ISBN 978-950-579-311-2. Posadas Argentina. 
González Suárez, E.; (2014). Proyecto Institucional: Formación de la Capacidad Científica de la Región Central de la Investigación Científica en la industria química y fermentativa. Univ. Central de las Villas. Villa Clara, Cuba.

González Suárez, E., Miño Valdés, J.E.; (2014). Aspectos de la Estrategia de Procesos para el aprovechamiento de la biomasa como fuente de productos químicos y biocombustibles. Editorial Universitaria de Misiones, ISBN. 978-950-579-339-6, Posadas Argentina.

González Suárez, E., Miño Valdés, J.E., (2015). Acciones para la correcta terminación y valoración de resultados en la industria química y fermentativa. Editorial Universitaria de Misiones, ISBN 978-950-579-379-2. Posadas, Argentina.

Miño Valdés, J.E., González Suárez, E., Herrera Garay J.L.; (2015). Estrategia Innovativa en el desarrollo de una tecnología para elaborar vino blanco con uvas no viníferas. Editorial Universitaria de Misiones. ISBN 978-950-579-369-3. Posadas Argentina.

Miño Valdés, J.E.; González Suárez, E. (2015). Vías para la gestión de conocimientos con apoyo de la comunidad científica para contribuir a la mitigación del impacto ambiental de la industria química y fermentativa. Editorial Universitaria de Misiones. ISBN 978950-579-368-6. Posadas, Argentina.

Waissbluth, M., (1994). Vinculación de las investigaciones Científicas y tecnológicas con las unidades productivas. Ciencia, Tecnología y Desarrollo: interrelaciones teóricas y metodológicas. Editorial Nueva Sociedad, pp.387-410. Santiago, Chile. 